\title{
Discovery of Meridian Entities and the Third Regulatory Framework
}

\author{
Xu Yueyuan \\ Hospital Management Office, Beijing University of Chinese Medicine, Beijing, China \\ Email address: \\ bzyylc@126.com, Xuyueyuan2010@sina.com \\ To cite this article: \\ Xu Yueyuan. Discovery of Meridian Entities and the Third Regulatory Framework. American Journal of Biomedical and Life Sciences. \\ Vol. 9, No. 5, 2021, pp. 230-235. doi: 10.11648/j.ajbls.20210905.11
}

Received: August 8, 2021; Accepted: August 31, 2021; Published: September 8, 2021

\begin{abstract}
Human diseases are the result of neuromodulation and humoral regulation disorders. However, the Chinese people have carried out acupuncture experiments on the body for thousands of years, and their curative effects have convinced them that the meridians and acupoint structure based on acupuncture and moxibustion is not a simple mechanism of nerve conduction and humoral regulation. This study adopts "Xu's pulse diagnosis Intangible Cultural Heritage Inheritance technology". According to "Lingshu - meridians", the pulse on the skin surface of the whole body was perceived. Pulse diagnosis found that there were longitudinal and transverse pulsating thin lines on the body surface. Then through the meridian pen to test the vertical and horizontal conduction path, acupuncture to verify the clinical effect, and local anatomy to confirm the existence of meridian and acupoint entities in the human body. Finally, it is determined that there are 31 pairs of transverse meridians and 13 pairs of longitudinal meridians in human epidermis and dermis. It is highly consistent with the meridian path of the twelve meridians, the odd meridians and the eight meridians of traditional Chinese medicine, and the position of acupoints is also highly consistent. According to the new development of skin anatomy, this paper expounds the physiological and pathological regulation mechanism of meridian structure, and determines that there is an artificial external regulation system based on vertical and horizontal meridian structure in human body.
\end{abstract}

Keywords: Meridians, Skin Pulsations, Acupoints, Acupuncture

\section{The Path of the Meridians on the Human Body Surface}

"Huangdi Neijing" is the earliest medical classic in TCM. The elaboration on meridians provides theoretical basis for Qihuang medicine and acupuncture. Cultural relics excavated in modern times, such as the "Book of Pulses", "Eleventh Meridians of Foot Arms", "Eleventh Meridians of Foot Arms and Moxibustion Vessels", " Fushui meridian wooden man " demonstrate all age of meridian structure. At present, the theory of viscera and acupuncture are widely used in clinical practice, However, there is still a lack of scientific basis for the study of meridian entities. Based on the careful study of Lingshu meridians, the author has practiced the "Xu pulse diagnosis intangible cultural heritage technology" [1] to detect human skin pulsation of the very layer of the human body's superficial skin area, combined the most advanced view in the anatomy of modern medicine, in order to prove the actual existence of the meridian entities. It is built on the superficial pulsation of the human body vertically and horizontally. The human epidermis has microcirculation of micro-vessels, venous anastomosis, and its micro-neural cutaneous branch management. The dermis has a vertical and horizontal network structure of $\mathrm{C}$ nerves, arterioles and veins. $\mathrm{C}$ nerves have no myelin. If they are adjacent or contacted with each other, there will be contact junction or gap junction. Then they will build a pulsating crisscross circuit with small arteries and veins. At the same time, it also provides anatomic support for the two-way conduction of channels, that is, Root knot phenomenon, and provides a cyclic connection mode for the construction of channels theory. [2]

The human body has 31 pairs of nerve segments, passing through the spinal nerves to each other. They contact the internal organs and terminate at the midline anterior to the abdomen. The parts under the control zone (body surface and internal organs) are interconnected by nerves. Acupuncture is selected to intervene along with the nerve segments for the 
viscera disease. It has the value of nerve regulation from the outside to the inside [3]. The Acupuncture on the twelve regular channels of the limbs all have this kind of neural regulation mechanism. 31 pairs of nerve and blood vessel segments send out dermal and epidermal branches to each other in the dermis, forming an overlapping connection across the segments [4], and a longitudinal route of channels with 13 pairs of pulsating fine-line sensations 31 [5], for example, the eight veins of the odd meridian in traditional Chinese medicine, and the longitudinal pulsation of the three yin and three yang channels of the foot. Due to 13 pairs of longitudinal connections across the nerve segments, the longitudinal-horizontal connection pattern of channels and collaterals is constructed. Clinically, the acupuncture of channels will result in the slow conduction mechanism of needle sensation, such as the left diseases are treated on the right side, the upper diseases are treated on the bottom, the anterior diseases are treated on the back, and the inner diseases are treated on the outside. If clinically acupuncture on the transverse channel is the fast conduction associated with nerve conduction, then acupuncture the longitudinal channel is the slow conduction of the $\mathrm{C}$ nerves across the segments. The slow conduction is the "main points" in the sentence "The puncture rising and falling, so as to know its main points" mentioned in the Huang Di Nei Jing. However, the absurd acupuncture or giant thorn effect emphasized by ancient acupuncture is more dependent on the trans-neuronal segmental effect.

The longitudinal channel of the skin across nerve segments is similar in function to the intrinsic basal bundle of the spinal cord, which runs through each segment of the spinal cord [6]. Of course, there are overlapping connections between the spinal cord segments. This overlapping connection to a certain extent ensures that the human body completes various complex reflex activities, completes the regulation and coordination function of the human body's trans-neural segmental conduction. Therefore, to some extent it maintains the partial sensation of the skin in the case of spinal cord transection or spinal cord shock.

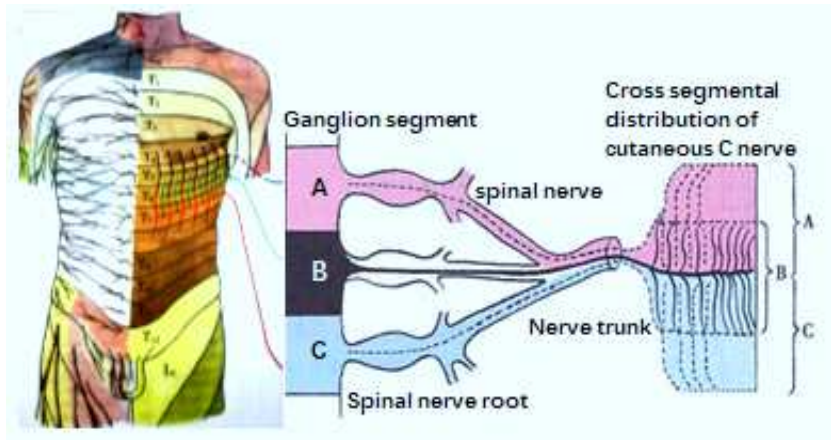

Figure 1. Cross-segmental connection of small $C$ nerves and blood vessels in the dermis.

The 13 pairs of longitudinal channels are issued by the blood vessels of 31 pairs of transverse spinal ganglion segments in the human body. The dermal branches of nerves form the longitudinal and transverse connections of human body. The crossing points of the longitudinal and transverse are the issuing points of nerves and blood vessels. Because it is perpendicular to the finger, and the sense cycle is always pulsating in the shape of small points. (Figure 1)

In short: meridian circulation is composed of 31 pairs of transverse meridians and 13 pairs of longitudinal meridians. Acupoints are the junction of vertical and horizontal meridians. The meridian line pulsation and acupoint pulsation shown in the anatomical diagram (2).

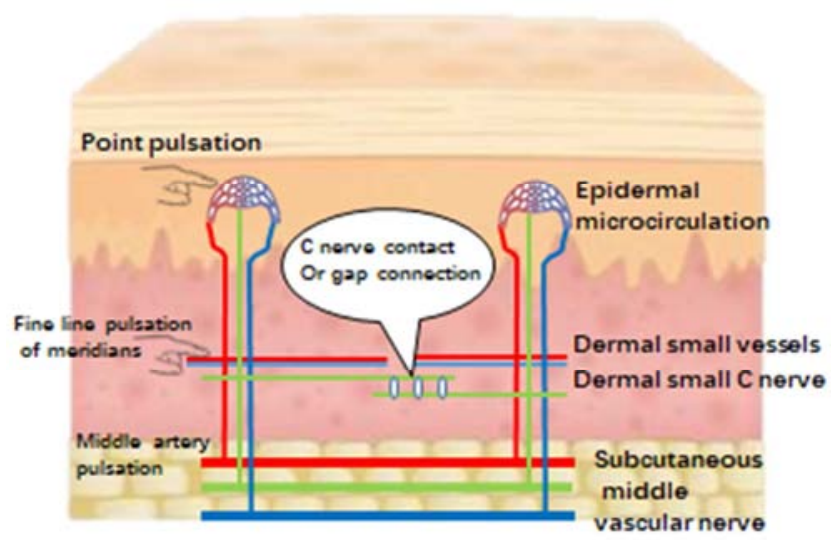

Figure 2. Channel, points pulsation.

\section{The Relationship Between Channels, Points, and Anatomy}

Figure 2 shows the acupuncture points with three tree-like structures: the arterial tree, venous tree, and nerve tree.

\subsection{Arterial Tree}

The subcutaneous or intermuscular middle artery (about $\varphi 2 \mathrm{~cm}$ ) branches into small arteries (about $\varphi 0,3-1 \mathrm{~mm}$ ) and walks in the dermis, and the small arteries walk toward the epidermis and branch into micro arteries (about $\varphi 0,3 \mathrm{~mm}$ ). Micro-arteries form a microcirculation. At the same time, some small branch blood vessels span adjacent blood vessels and nerve segments to form overlapping lines, which is also the basic structure for the formation of thin line-like pulsations and punctate pulsations. The blood flow of the arterioles branched from the inside to the outside. Observed under a low-power microscope, it looks like a tree [7].

\subsection{Vein Tree}

Veins and arteries accompany blood flow from the outside to the inside.

\subsection{Nerve Tree}

cutaneous branch of $\mathrm{C}$ nerve in epidermis and dermis. In particular, the extensive connection of the cutaneous branch of $\mathrm{C}$ nerve in the adjacent segments of dermis and epidermis, accompanied by arteries and veins, constitutes a series of meridians connection phenomena of vertical and horizontal 
skin and two-way slow conduction, which provides anatomical support for acupuncture and moxibustion and even Meridians Theory.

\subsection{Transverse Meridians}

Specifically refers to the transverse small dermal vessels and cutaneous branches of $\mathrm{C}$ nerve. There are 31 pairs on the left and right sides of the human body, which pulsating like a thin line when feeling. It is mainly found in three yin and three yang of hands and feet.

\subsection{Collaterals}

Refer to the structure of longitudinal small blood vessels and $\mathrm{C}$ nerves in the dermis. It is emitted from the transverse meridians and has longitudinal linear pulsations when feeling, for example, the eight odd meridians.

\subsection{Meridians}

Specifically refers to the operation mode of Qi and blood built between vertical and horizontal meridians.

\subsection{Acupoints}

They are the connecting branches of epidermal, dermal and subcutaneous blood vessels and nerves, which form a wide connection by forming crisscross small blood vessels and nerves in the dermis. Crisscross points are the positions of acupoints.

Due to the association of blood vessels and nerves, Qi Huang school in the Warring States period found vertical and horizontal pulse lines on the body surface through pulse diagnosis. Select the distal acupuncture along the meridian to achieve clinical curative effect and form the meridian medicine represented by Bian que. In the Yellow Emperor's internal classic written by the Qi Huang school in the late Han Dynasty, the meridian phenomenon [8] was further explored and the circulation theory was constructed. The small palpable pulsations on the meridian line were named acupoints. Figure 3 is an anatomical view of the pulsation of the hand Taiyin.

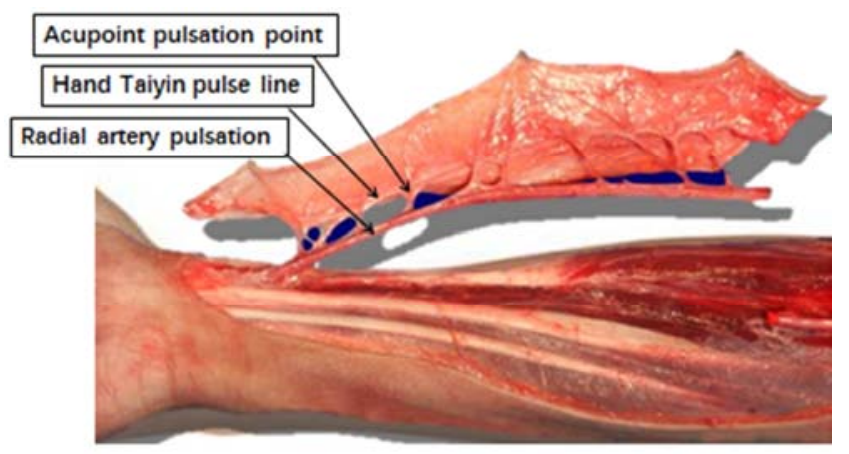

Figure 3. An anatomical diagram of hand Taiyin pulsation.

\section{Study on Meridian Mechanism}

Acupoints are densely distributed on human skin and connected with each other through $\mathrm{C}$ nerve in dermis to form an array similar to "domino effect", similar to hip-hop dance hand in hand: microcirculation units contract or expand under the transmission of $\mathrm{C}$ nerve information, so as to regulate local physiology and pathology, which constitutes an artificial readjustment functional structure, It has the effect of relieving pain by regulating and controlling the disorders of nerves and body fluids in the human body. On the other hand, "three trees" form an umbrella shaped uplift structure on the skin, expanding the touch surface. This physiological structure can ensure the realization of skin excretion and absorption function, and increase skin sensitivity. Light, sound, electricity and other stimuli can produce response [9], and maintain the transmission of stimulation effect without attenuation [10]. Of course, the meridians are not anatomically direct channels, but the phenomenon of vasoconstriction and resonance formed by the mutual contact and gap connection of demyelinated nerves. (Figure 4)

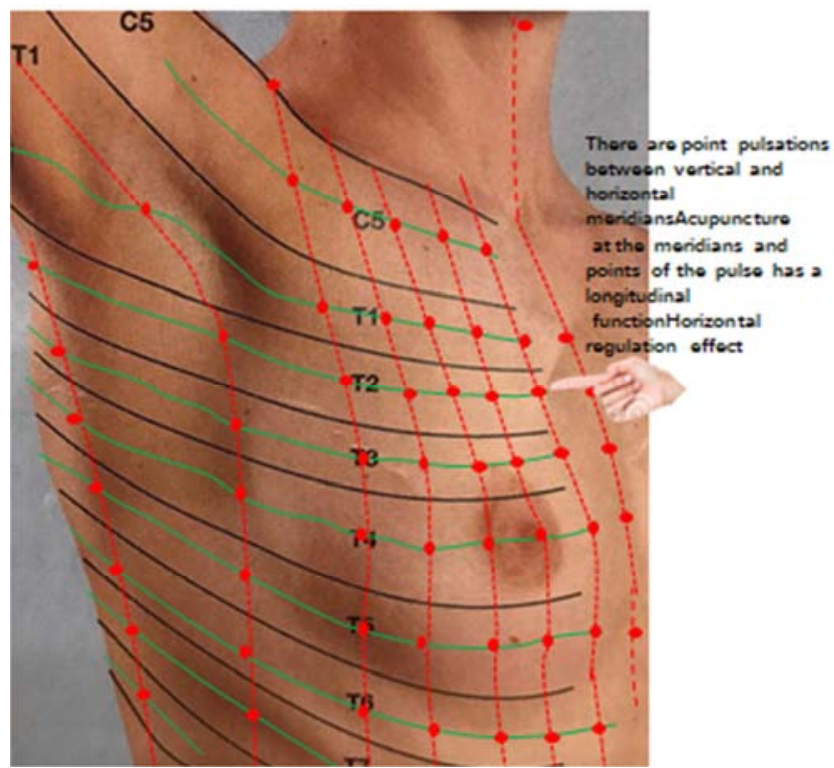

Figure 4. The acupoints at the junction of the vertical and horizontal meridians and acupoints of the human body.

Note: There are a total of 26 longitudinal red lines before and after it. The ancients named it the three yin and three yang parts of the foot and Eight veins of the odd meridian. There were 31 pairs of transverse yellow lines. In ancient times, the cutaneous branches of the nerve and blood vessel segments from Neck 4 to Chest 1 were named after the three yang and three yins of hands. The black line indicates the segmental boundary on the plane of nerves and blood vessels.

The cutaneous branches of the $\mathrm{C}$ nerve in the meridians are connected with each other and pass vertically and horizontally through the upper and lower, left and right, front and back, inside and outside of the human body, maintaining the same relationship with the nerve trunk as water and wave. Therefore, the meridians have dual physiological regulation effects on nerves and body fluids. At the same time, when the mechanism of human self-control is disordered, the meridians will be artificially re regulated. Therefore, percutaneous acupuncture, moxibustion, scraping and 
massage are the methods to treat diseases, not the auxiliary medical model.

\subsection{Meridian Sensitization}

Meridians have the effect of independent and manual intervention, because acupoints with "effector" nature are densely arranged on the meridian line and support the meridian phenomenon [11]. This structure constitutes the third regulatory (artificial re regulation) framework in addition to neural and humoral regulation. Visceral or body surface diseases can cause $\mathrm{C}$ nerve sensitization in corresponding areas and cause various body surface pain [12] As long as 31 pairs of spinal nerves and 13 pairs of longitudinal choroids pass through the sensitized area of $\mathrm{C}$ nerve, they will have a regulatory or sensitizing effect on the sensitized area, and the sensitized $\mathrm{C}$ nerve will have a meridian effect. (Figure 5)

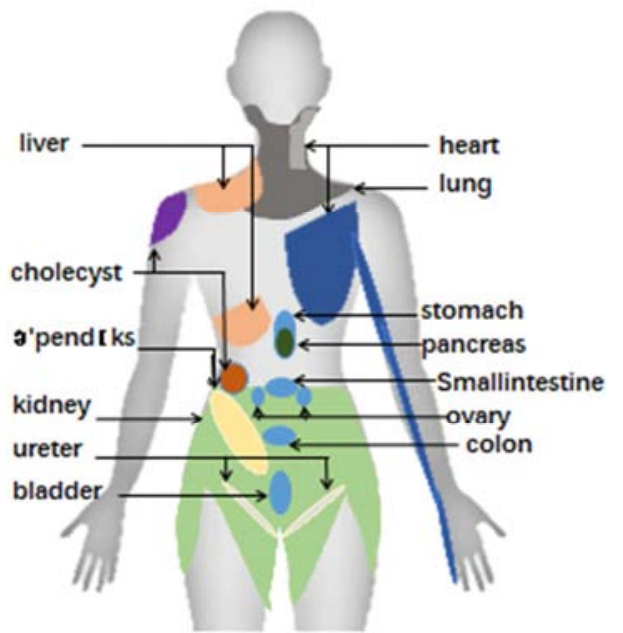

Figure 5. Body surface referred pain and C nerve sensitization.

\subsection{Trans Nerve Segment Conduction Acupuncture Test}

Stimulation effect and meridian conduction are related to human nerves, but the more important performance is trans nerve segment conduction. The patient felt pain in the gallbladder area, which was caused by nerve c-sensitization in the gallbladder area. Acupuncture at Yanglingquan was selected to immediately eliminate the pain in the gallbladder area [13]. This also shows that the artificial intervention of distal acupoints can improve the inflammatory response in the gallbladder area. Acupuncture at Zusanli is beneficial to the treatment of gastrointestinal discomfort or chronic gastritis. Repeated acupuncture is effective [14], which is related to the connection of zuyangming stomach meridian points. For chronic pharyngitis, acupuncture at Shaoshang point makes the patient feel comfortable in the pharynx. Therefore, acupuncture at Shaoshang point with injection needle will lead to bleeding and immediate relief of pharyngeal congestion [15]. The transcutaneous nerve endings of hand Yangming pass through segments, and the nerve connection is composed of cervical segments 6-7 related to pharynx.

\subsection{Pre-or Post-ganglionic Inhibition Test}

Acupuncturing at Linggu acupoint has a definite curative effect on non-prolapsed intervertebral disc protrusion and bulge [16]. The principal of effectiveness is that acupuncturing at this acupoint can relax the spasmodic lumbar muscles and remove the inducement for intervertebral disc protrusion or bulge. After acupuncturing at Mingyan acupoint, the patient's eye will be immediately comfortable and bright because both spinal nerves 6-7 and the thoracic cutaneous nerve one can regulate the eyes through the sympathetic ganglia.

\subsection{Direct Stimulation of Organ Nerve Trunks or Ganglia}

Deep insertion from Huatuo Jiaji acupoint to nerve shell is effective for the clinical treatment of visceral diseases. Select Huatuo Jiaji acupoint in the sixth thoracic vertebra and gently tap the outer membrane of the nerve trunk with the needle can immediately solve the symptoms such as stomachache, poor appetite, dyspepsia, and gastrointestinal discomfort caused by chemotherapy. Acupuncturing at sphenomaxillary ganglia: an oblique insertion of $55 \mathrm{~mm}$ along the direction of contralateral eye of the sphenomaxillary canal, and touching the sphenomaxillary ganglia, has a positive effect on allergic rhinitis. All these indicate that acupuncturing at the nerve has a curative effect on disease [17]. Besides the nerving effect, the effects of meridians and acupoints are also admixed with the contraction-release linkage of the lesion's blood circulation.

\subsection{Euphoriant Effect}

The stretching of meridians and collaterals and the filling of blood often make people feel euphoric and relaxed in muscles. For example, shallow punture of $\mathrm{Hegu}$ and Taichong acupoints with fine needles, or shallow punture of the upper and lower edges of the navel can make patients keep warm and have a rest. Patients often feel the euphoria of ethereal lightness. In fact, this is the vasodilation effect triggered by the stimulation of the parasympathetic nerve by acupuncture [18].

\subsection{Temperature Control Effect or Antihypertensive Effect}

For patients with high fever, we choose Quchi, chize, Shaohai and contralateral Hegu points for acupuncture, which generally reduces fever within 20 minutes. Hypertension: similarly, selecting the above acupoints can also reduce blood pressure. Its mechanism is the vasodilation and perspiration function of autonomic nerve [19].

\subsection{Regulating Willpower Effect}

Randomly select patients with depression, deeply stab Yongquan point through Baihui Point and sinking Valley (EX-B2), rest for 30 minutes, and the symptoms are significantly relieved [20]. Repeated treatment can significantly alleviate the disease. 


\subsection{Effects of Endocrine Intervention}

If the cutaneous branches of 6-9 costal nerves, the bladder meridian, Yangwei and Yangqiao acupoints were directly punctured, the patient's breast distending pain would disappear immediately, and the texture would become soft.

\subsection{Direct Transection of Meridians in the Focus Area Has a Wide Range of Clinical Applications}

For example, western medicine chooses nerve root ablation to treat intractable diseases, such as sequelae, trigeminal neuralgia, herpes zoster and neurogenic migraine. In traditional Chinese medicine, the small knife needle cutting operation of selecting the meridian line of the diseased part can achieve the purpose of immediate pain relief, and the clinical effect is twice the result with half the effort.

\section{Two Way Conduction Test}

When the pulse feels fish seam, select the meridian pen to shock fish seam. The stimulation can be transmitted to Shaoshang, great gulf and sometimes as far as supreme cave. (Figure 6)

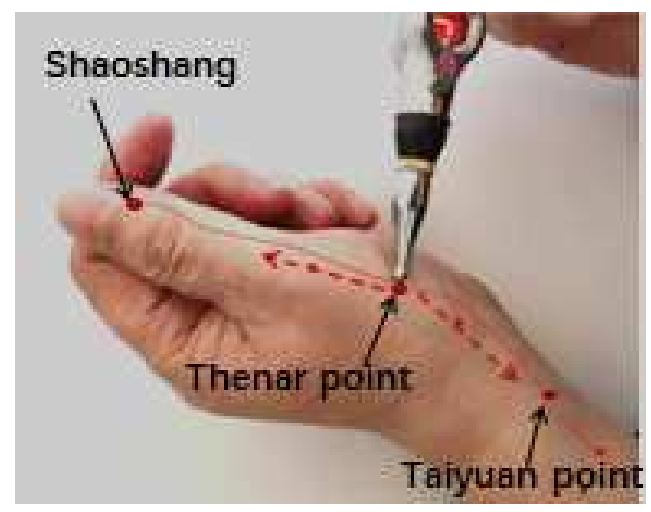

Figure 6. Two-way sensory transmission occurs when the meridian pen shocks Yuji.

It is not necessary to select sensitive people in meridians and collaterals for this test. This result exists in normal people as well. Mystifying the meridian phenomenon is a scientific lie. The reason why there are many hypotheses about meridian entities is that we have not carefully studied the classics of traditional Chinese medicine and lacked the techniques of meridian sensation and its inheritance.

\section{Discussion}

By using the Xu Shi propagated sensation along meridian technique, the author has discovered the properties of propagated sensation along meridian and their distribution in human skin, revealing the essence and construction mode of meridians and even their meridians. The meridian and collateral, the micro and small unmyelinated nerves and vascular structure that surround the whole body, communicate up and down, and connect the inside and outside, are still an artificial external regulation system with no clear understanding of anatomy and physiology or visible effects. Besides, it also verifies the scientificity of the arguments in Qihuang Medicine and even TCM acupuncture.

\section{Conclusion}

31 pairs of transverse meridians and 13 pairs of longitudinal collaterals are distributed in the human dermis, and the crisscross points are the acupoints. In ancient Chinese medicine, the meridian theory was constructed based on the external pulsation of the hand sensation in the user's hand to guide clinical application such as acupuncture. Because it still acts on the artificial re-regulation when the regulation of nerves and body fluids is in disorder, and it is characterized by simplicity, rapidness, and immediate effect in the clinic, we named it the third regulation structure and system in the human body.

Limited to the length, the specific new meridian diagram would publish again. Subsequently, the author will publish a series of related studies on meridians and their acupoints one after another in order to initiate further studies. Meanwhile, I look forward to receiving the correction and participation of all scholars.

\section{References}

[1] The "intangible cultural heritage technique of the $\mathrm{Xu}$ pulse diagnosis": pulse diagnosis "shape", "qi" and disease \% pulse diagnosis "shape", "qi" and diseases [J]. Chinese Journal of Traditional Chinese Medicine, 2019, 034 (001): 29-33.

[2] Wang Kemo (1992) physiological function and physiological scientific progress of unmyelinated nerve fibers. 23. 126-130.

[3] Pan Satellite. Neurobiological mechanism of acupuncture and moxibustion. Chinese Journal of Traditional Chinese Medicine 033. 010 (2018: 4281-4297.

[4] Cross-sectional information transmission between sensory nerve endings in rats. Journal of Xi 'a Medical University (1996).

[5] Xu Yueyuan and Zhao Pengfei. "Lung Meridian of Hand Taiyin discovered through pulse diagnosis." Chinese Journal of Traditional Chinese Medicine 35. 8: 4. (2020).

[6] Shen Li, Xu Luxi, Ma Weiyi. The connection between the initial cells of the descending spinal fasciculus proprius and the dorsal lateral funiculus in the spinal cord of rats $[\mathrm{J}]$. acta anatomica sinica, 1988 (03): 28-33+117.

[7] Baishu Ling, Li Ji. Experimental morphological study on the vascular tree of skin microcirculation (5) $[\mathrm{J}]$. Chinese Journal of Burns and Ulcers (1): 5-8.

[8] The terminal phenomenon of meridians: TCM Bulletin 2009 No. 6, pp. 16-16, 1 page in total.

[9] Li Ding. Exploration of meridians and collaterals for 60 years discussion with Mr. Liu Chengzhong on the research of meridian phenomena $[\mathrm{J}]$. Culture of Traditional Chinese Medicine, 2012 (2): 36-38. 
[10] Li Dingzhong, Fu Songtao, Li Xiuzhang. Overview of meridian research and empirical study of its existence-one of the theoretical and clinical application studies on meridian [J]. Chinese Acupuncture and Moxibustion, 2004, 024 (011): 773-778.

[11] Sharla Cheung. The essence of meridians [C]// Summer Summit of World Congress of Traditional Chinese Medicine and "the belt and road initiative" International Symposium on Development of Traditional Chinese Medicine. 2015.

[12] Li Chunchang. What is "involved pain"? How did it happen? [J]. Chinese Community Physicians, 2010 (04): 2.

[13] Bin Luo. Immediate effect observation of acupuncture at Yanglingquan (GB 34) in relieving biliary colic [J]. Modern Medicine and Health Research, 2018 (000) 014.

[14] Zhu Yuanyuan. Application of acupuncture in the treatment of chronic gastritis [J]. Chinese Journal of Traditional Chinese Medicine, 2015 (06): 1527-1529.

[15] Xiang Ping. Shaoshang acupoint blood-letting puncture treatment of acute pharyngitis in 120 cases [J]. Journal of practical traditional Chinese medicine, 2012, 28 (002): 133-133.

[16] Ji Bingjin. Acupuncture treatment of lumbar disc herniation research progress $[\mathrm{J}]$. Journal of practical traditional Chinese medicine, (2012) 07-607-02.

[17] XU Zuo-shi. Pulse electroacupuncture stimulation of sphenomaxillary ganglia in 104 cases of allergic rhinitis [J]. Fujian Medical Journal, 1995 (3).

[18] Wang Wenjin. Ancient literature study on the primary application of acupuncture at Taichong (LR3) [D]. Shandong University of Traditional Chinese Medicine, 2012.

[19] Fan Ran, Gao Ying, et al. Research progress on the mechanism of acupuncture at Quchi (LI 11) in treating hypertension. Chinese Electronic Journal of Acupuncture and Moxibustion. 2015. Vol. 4 No. 1

[20] Zhou Mingqian, Xuan Lihua. Acupuncture at Taichong (LR 3) and through Yongquan (GB 34) for treating 19 cases of insomnia $[\mathrm{J}]$. Shanghai Journal of Acupuncture and Moxibustion, 2011, 30 (005): 328-328. 